\title{
DARPA MMW system programs and how they drive can compound semiconductor technology needs
}

\author{
H. Bruce Wallace, Life Fellow \\ Defense Advanced research Projects Agency, 675 N. Randolph St, Arlington, VA
}

\begin{abstract}
DARPA has been investing in the development of compound semiconductors significantly since the MMIC program was initiated in the 1980 's. This has resulted in the maturation of MMW systems for use in both commercial and military enterprises. Subsequently, Silicon technologies have matured significantly and are supplanting them in a variety of uses, such as automotive radar and indoor communications. This plenary presentation will discuss several DARPA programs that are dependent upon extending the performance of compound semiconductors beyond what Silicon appears to be able to achieve.
\end{abstract}

Index Terms - phased array, SAR, MMW, InP, GaN, Silicon, MMIC

\section{INTRODUCTION}

DARPA has been investing in the development of compound semiconductors significantly since the MMIC program was initiated in the 1980's. This has resulted in the maturation of MMW systems for use in both commercial and military enterprises. Subsequently, Silicon technologies have matured significantly and are supplanting them in a variety of uses, such as automotive radar and indoor communications. This paper will discuss several DARPA programs that are dependent upon extending the performance of compound semiconductors beyond what Silicon appears to be able to achieve.

\section{MATERIAL TO BE COVERED}

Several programs in the DARPA Strategic Technology office will be covered in this plenary presentation and will be summarized in the final paper. They include Mobile Hotspots, 100G, Multifunction Radio Frequency (MFRF), and Video Synthetic Aperture (ViSAR), all of which operate at frequencies above $70 \mathrm{GHz}$. Each program has its own unique characteristics which demand a different compound semiconductor MMIC investment. These system requirements, the current state-of-the-art, and the ultimate needs will be discussed as well as how these system requirements limit the use of silicon approaches to solving transmitter, receiver, and aperture needs.

\section{OVERVIEW OF DARPA MMW SYSTEM PROGRAMS}

\section{A. Mobile hotspots}

The goal of the Mobile Hotspots (MHS) program is to design, build, and test a self-organizing, scalable mobile communications backhaul network with the capacity and range needed to connect remote and mobile warfighters with forward operation bases (FOBs), higher-echelon tactical operations centers (TOCs), remote intelligence, surveillance, and reconnaissance (ISR) sources, and fixed communications infrastructure. The program will develop the mobile hotspots capability as a pod-based system carried on UAVs. The electronics being developed are leveraging E-Band technology to provide for $1 \mathrm{~GB} / \mathrm{sec}$ links over tactically relevant distances and provide for air to ground links at microwave frequencies or higher data rates in E-Band. These requirements drive us to develop high efficiency power combined amplifiers for the long haul and very compact ground units.

\section{B. $100 G$}

The goal of the $100 \mathrm{~Gb} / \mathrm{s}$ RF Backbone (100G) program is to design, build, and test an airborne based RF communications link with fiber-optic equivalent capacity and long reach capable of propagating through clouds and providing high availability. The system will provide 100 $\mathrm{Gb} / \mathrm{s}$ capacity at ranges of $200 \mathrm{~km}$ for air-to-air links and $100 \mathrm{~km}$ for air-to-ground links when installed in a highaltitude (e.g. 60,000 ft.) aerial platform. The system will provide an all-weather (cloud, rain, and fog) capability while maintaining tactically-relevant throughput and link ranges. In addition to developing the required E-Band power sources and receiver, the program will invest in approaches for high-order modulation and spatial multiplexing. Some early efforts have demonstrated an Indium Phosphide (InP) modulator providing direct RF signal modulation at data rates in excess of $25 \mathrm{~Gb} / \mathrm{s}$ within a $5 \mathrm{GHz}$ bandwidth for spectral efficiencies exceeding 5 $\mathrm{b} / \mathrm{s} / \mathrm{Hz}$. The use of Nyquist Cyclic Modulation with 32QAM and 64-QAM demonstrated significant reduction in the power backoff (from saturation) over other common modulation schemes. It should be noted that the 
computational complexity of the millimeter-wave MIMO processes required between 100 and 2500 operations per information bit. As $100 \mathrm{G}$ moves to full rate, efficiency in spatial multiplexing computations is required to achieve program goals for throughput as well as size, weight, and power (SWaP).

\section{C. $M F R F$}

The DARPA Multifunction RF program (MFRF) grew out of the need to provide a capability to provide for safe landings, obstacle a voidance, and collision avoidance in a manner that will not adversely affect the SWAP of helicopter platforms. In order to achieve this capability; it is necessary for this system to incorporate functions that are currently executed by lower frequency radars operating at $\mathrm{Ka}$ and $\mathrm{Ku}$-band. Resolution requirements drove the system to operate at as high a frequency as possible within the limitations created by atmospheric propagation and available semiconductor technology. Because of the complexity of the RF functions, the preferred approach was to build a tiled phased array using IBM 8HP beamformers. The resulting beamformer comprised an $8 \times 8$ array with dual polarization, attenuation and phase control at each element, and up conversion and down conversion to an IF that could readily distributed across the array which was composed of approximately 25000 elements. While the beamformer design was successful, the integration into a workable tile has proved difficult and the losses associated with integration reduced the maximum range of operation. Improved beamformer technology is being actively pursued to overcome these losses to meet the notional system requirements.

\section{ViSAR}

Close-in air-support aircraft, such as the AC-130 gunship, typically use infrared targeting sensors to engage moving ground targets such as vehicles and dismounts. Since infrared sensors are not able to image through the clouds, close-in air support is currently limited to clear weather conditions. In addition, once engagements commence, infrared sensors are unable to image through the dust caused by explosions and incoming rounds.

We're addressing this shortfall by developing a highfrequency airborne radar that can image the ground through clouds and dust at a sufficiently high resolution and frame rate to support engaging maneuvering targets. For Synthetic Aperture Radar (SAR) with a given resolution, the frame rate is proportional to the frequency, thus moving to a higher frequency provides a higher frame rate (and also a lower latency). Moving to higher frequency also results in a smaller antenna size. On the other hand, moving to a higher frequency also incurs higher atmospheric losses, particularly in the presence of clouds. After going through all these trades, we decided to operate in the $231.5-235 \mathrm{GHz}$ band. This is an approved radiolocation band (and thus we can get permission to radiate) and provides the desired frame rate at acceptable atmospheric losses.

We're building a prototype ViSAR system and we plan to demonstrate it in over-the-air in flight tests in 2016. The ViSAR system consists of a $233 \mathrm{GHz}$ front- end that up converts from and down converts to a back-end that's built from conventional microwave radar. The $233 \mathrm{GHz}$ portion will be installed in a tactical gimbal, the MultiSpectral Targeting System-B (MTS-B). The ViSAR antenna system includes five small antenna horns, one horn for transmit and four receive horns. There are four receivers (one for each receive antenna) each with an $A / D$ converter. Signal-processing algorithms process the data to generate high-resolution, ground-focused SAR images and detect and relocate moving targets. The imagery is mapped into an angle/angle display similar to what the AC-130 operators are familiar with.

\section{E. ASTIR}

The goal of the Advanced Scanning Technology for Imaging Radars (ASTIR) program is to demonstrate a new imaging radar architecture using an electronic subreflector to produce a more readily available, cost effective sensor solution that does not require platform or target motion as in SAR or ISAR. The ASTIR concept will minimize system complexity by using a compound antenna with an electronic sub-reflector and a single transmit/receive chain. The sub-reflector would work in concert with a large primary aperture that would define the angular resolution of the radar. Numerous techniques are being investigated to provide the scanning including liquid crystals, diode phase shifters, and vanadium oxide switches. Integration of the diodes into a control structure is the most challenging engineering effort but the performance of the diodes themselves of the frequencies of interest (70-700 GHz) will ultimately limit the system performance.

\section{COMPOUND SEMICONDUCTOR INVESTMENTS TO ADVANCE CAPABILITIES}

The common needs from the compound semiconductor community go beyond the typical requirements for lower noise, higher gain, more power, and improved power amplifier efficiency that MMIC developments usually strive to address. With Silicon technology we can yield a 
very high level of integration in the beamformer. When building large arrays, as in the MFRF program, transmit powers less than $10 \mathrm{~mW}$ are required per element. Current limitations in the beamformers are the power consumed by all of signal control functions. One beamformer circuit can provide 64 radiating elements but draws upwards of $8 \mathrm{~W}$. The array being developed has 320 tiles which thus adds up approximately $3 \mathrm{~kW}$ of power in order to radiate approximately $8 \mathrm{~W}$ of peak power. Our program is currently looking for alternatives to the IBM 8HP process for the beamformers in an attempt to drop the power consumption by half. But even with this efficiency improvement, the integration of the beamformers into working tiles that can radiate reduces the figure of merit (FOM - transmitter power minus noise figure) below acceptable limits. The most likely solution is to integrate InP into the array to improve transmitter power by $6 \mathrm{~dB}$ and noise figure by $3 \mathrm{~dB}$. Various techniques for accomplishing this have been examined based on the efforts in the DARPA SMART and COSMOS[7] programs.

When power higher powers are needed for systems such as MFRF, 100G, and ViSAR, power combining of state of the art MMICs in GaN or InP has been exploited to varying degrees of success. Waveguide power combining has been used to combine up to 32 amplifiers in at 235 $\mathrm{GHz}[8]$ but the resulting total power is limited due to losses in the transitions from the MMICS to the waveguide modes. The literature has described multiple techniques for power combining but the resulting measured losses and mismatches for the output transitions can severely impact both the output power and bandwidth. We have also noted that the use of high gain and wideband MMICs and result in significant AM noise which will desensitize receivers unless herculean efforts are made to isolate them from the transmitter. The radar systems we develop are typically time gated to reduce the isolation requirements. This requires either the modulating the bias, which has turnon/turnoff limitations, or insertion of a switch between the MMIC, which reduces gain. The development of narrower band MMICs with integrated switching circuitry would solve these problems. While narrowing the bandwidth may seem to reduce the possibility of multiple uses, power amplifiers are limited to operating in a few well defined frequency bands.

These two major issues, integration and MMIC response, drive our systems to greater complexity and negatively impact the SWAP that we are striving for.

\section{$\mathrm{V}:$ CONCLUSIONS}

DARPA has been investing in the development of compound semiconductors significantly since the MMIC program was initiated in the 1980's. This has resulted in the maturation of MMW systems for use in both commercial and military enterprises. Subsequently, Silicon technologies have matured significantly and are supplanting them in a variety of uses, such as automotive radar and indoor communications. This paper will discuss several DARPA programs that are dependent upon extending the performance of compound semiconductors beyond what Silicon appears to be able to achieve.

\section{REFERENCES}

[1] DARPA BAA 12-23, www.fbo.gov

[2] DARPA BAA 15-22, www.fbo.gov

[3] DARPA BAA 11-46, www.fbo.gov

[4] H. Bruce Wallace, "DVE - Beyond the landing problem," SPIE Defense and Security Symposium, 5 - 9 May 2014

[5] DARPA BAA 13-06, www.fbo.gov

[6] DARPA BAA 14-53, www.fbo.gov

[7] S. Raman, T. Chang, C, Dohrman, M. Rosker, "The DARPA COSMOS program: The convergence of InP and Silicon CMOS technologies for high-performance mixed-signal,” 2010 International Conference on Indium Phosphide \& Related Materials (IPRM), May 31 2010-June 42010

[8] D. Gritters, K. Brown, E. Ko, Z. Griffith, M. Urteaga, "200$260 \mathrm{GHz}$ Solid State Amplifier with $700 \mathrm{~mW}$ of Output Power," Microwave Symposium Digest (MTT), 2015 IEEE MTT-S International, 23-28 May 2010 